\title{
Regioselective ring opening of epoxides by chelated amino acid esters enolates
}

\author{
Uli Kazmaier* and Ameer F. Zahoor \\ Institut für Organische Chemie, Universität des Saarlandes, D-66123 Saarbrücken, Germany \\ E-mail: u.kazmaier@mx.uni-saarland.de
}

Dedicated to Professor Siegfried Blechert on the occasion of his $65^{\text {th }}$ birthday

\begin{abstract}
Chelated enolates of $\alpha$-amino acids and peptides are suitable nucleophiles for the regioselective ring opening of epoxides. Depending on the substitution pattern, the reaction proceeds either in a $\mathrm{S}_{\mathrm{N}} 1$-type (arylepoxides) or a $\mathrm{S}_{\mathrm{N}} 2$-type (alkylepoxides) fashion, giving rise to $\gamma$-hydroxy $\alpha$-amino acids.
\end{abstract}

Keywords: Amino acids, chelates, epoxides, epoxide opening, peptide modifications

\section{Introduction}

Epoxides are very versatile building blocks in organic synthesis, which can be opened up with a wide range of nucleophiles. ${ }^{1}$ Elegant ring opening cascades found interesting applications in natural product synthesis, e.g. for macrocyclic polyethers. ${ }^{2}$ To apply this protocol also to peptidic natural products, suitable amino acid and / or peptide nucleophiles are required. The epoxide opening with glycine enolates should give rise to $\gamma$-hydroxy $\alpha$-amino acids, a structural motif widespread found in nature, e.g. in the glidobactins, ${ }^{3}$ arborcandins ${ }^{4}$ or the biphenomycins. ${ }^{5}$ Suitable C-nucleophiles are either isocyanoacetates, ${ }^{6}$ aminomalonates ${ }^{7}$ or imino esters. ${ }^{8}$ With chiral modified nucleophiles the stereochemical outcome of the reaction can be controlled. ${ }^{9}$ Berkowitz et al. reported the ring opening of ethylene oxide with various $\alpha$-amino acid ester enolates. ${ }^{10}$ Very recently, Crousse et al. reported the aminolysis of epoxides using $\alpha$-amino acid and peptide esters. ${ }^{11}$

\section{Results and Discussion}

Our group is also involved in amino acid and peptide synthesis, investigating reactions of chelated $\alpha$-amino acid ester enolates. ${ }^{12}$ These enolates not only show a higher stability compared to non-chelated enolates, but also a higher selectivity in a wide range of reactions such as aldol ${ }^{13}$ 
and Michael additions, ${ }^{14}$ as well as transition metal catalyzed allylic alkylations. ${ }^{15}$ These reactions are not limited to amino acid enolates but can also be carried out with peptides, ${ }^{16}$ while in several cases the stereochemical outcome of the reaction can be controlled by the stereogenic centers of the peptide chain. ${ }^{17}$ To enlarge the synthetic potential of these chelated enolates we were interested to see if these enolates can also be used for regioselective epoxide openings. In general, a $\mathrm{S}_{\mathrm{N}}$ 2-type mechanism is discussed ${ }^{1}$ resulting in an attack at the sterically least hindered position. This is reasonable for alkyl-substituted epoxides. $\mathrm{Li}^{18}$ and Ramachandran ${ }^{19}$ et al. investigated the opening of arylepoxides with allenoates and observed also a selective $\mathrm{S}_{\mathrm{N}} 2$-attack at the terminal epoxide position, even in the presence of Lewis acids. This is also true for $\alpha$-metalated carboxylic acids, ${ }^{20}$ while with malonates either $\mathrm{S}_{\mathrm{N}} 1$-type products are obtained or mixtures of both isomers. ${ }^{21}$

Therefore, we investigated the reaction of both, alkyl- and aryl-substituted epoxides 1 (Table 1). As nucleophile we used the enolate of TFA-protected $t$-butyl glycinate, which gave excellent results e.g. in Michael additions or allylic alkylations. $\mathrm{ZnCl}_{2}$ was used for chelation of the enolate and $\mathrm{BF}_{3} \cdot \mathrm{OEt}_{2}$ for the activation of the epoxide. The epoxide was used in slight excess (1.5 equiv.) to allow a complete conversion. In principle, two different products ( 2 and 3 ) can be formed. 2 is the preferred product of a $S_{N} 2$-type ring opening, while 3 should be found under $\mathrm{S}_{\mathrm{N}} 1$-conditions.

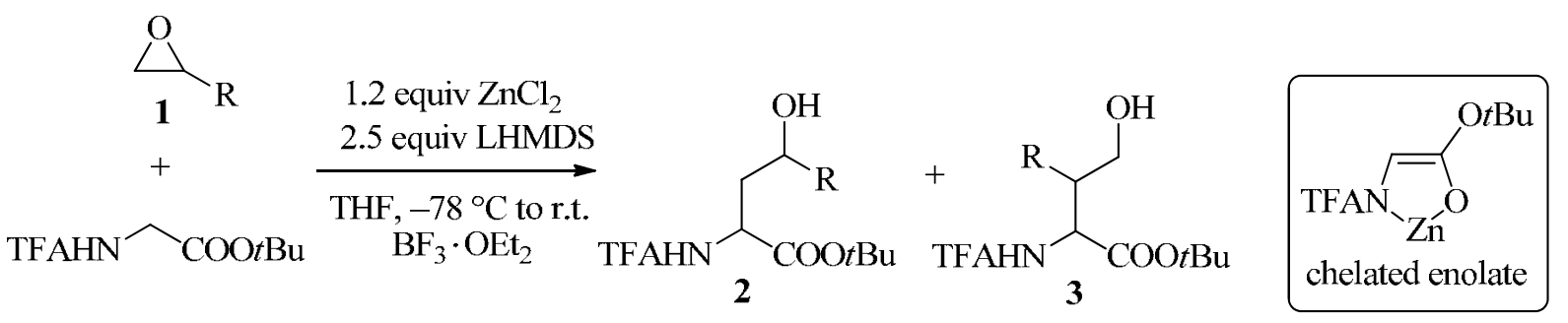

Table 1. Regioselective epoxide opening with chelated amino acid ester enolates

\begin{tabular}{cccccc}
\hline Entry & Epoxide & $\mathrm{R}$ & Product & Yield (\%) & Diast.-ratio \\
\hline 1 & $\mathbf{1 a}$ & $\mathrm{CH}_{3}$ & $\mathbf{2 a}$ & 92 & $68: 32$ \\
2 & $\mathbf{1 b}$ & $\mathrm{C}_{2} \mathrm{H}_{5}$ & $\mathbf{2 b}$ & 88 & $68: 32$ \\
3 & $\mathbf{1 c}$ & $\mathrm{C}_{4} \mathrm{H}_{9}$ & $\mathbf{2 c}$ & 86 & $66: 34$ \\
4 & $\mathbf{1 d}$ & $\mathrm{ClCH}_{2}$ & $\mathbf{2 d}$ & 74 & $56: 44$ \\
5 & $\mathbf{1 e}$ & $\mathrm{CH}_{2}=\mathrm{CH}\left(\mathrm{CH}_{2}\right)_{3}$ & $\mathbf{2 e}$ & 87 & $65: 35$ \\
6 & $\mathbf{1 f}$ & $\mathrm{C}_{6} \mathrm{H}_{5} \mathrm{OCH}_{2}$ & $\mathbf{2 f}$ & 86 & $56: 44$ \\
7 & $\mathbf{1 g}$ & $p-\mathrm{CH}_{3} \mathrm{C}_{6} \mathrm{H}_{4} \mathrm{OCH}_{2}$ & $\mathbf{2 g}$ & 81 & $55: 45$ \\
8 & $\mathbf{1 h}$ & $p-\mathrm{CH}_{3} \mathrm{OC}_{6} \mathrm{H}_{4} \mathrm{OCH}_{2}$ & $\mathbf{2 h}$ & 85 & $60: 40$ \\
9 & $\mathbf{1 i}$ & $\mathrm{C}_{6} \mathrm{H}_{5}$ & $3 \mathbf{i}$ & 67 & $75: 25$ \\
\hline
\end{tabular}


Table 1. Continued

\begin{tabular}{cccccc}
\hline Entry & Epoxide & $\mathrm{R}$ & Product & Yield (\%) & Diast.-ratio \\
\hline 10 & $\mathbf{1 k}$ & $p-\mathrm{CH}_{3} \mathrm{OC}_{6} \mathrm{H}_{4}$ & $3 \mathbf{k}$ & 71 & $67: 33$ \\
11 & $\mathbf{1 l}$ & $o-\mathrm{ClC}_{6} \mathrm{H}_{4}$ & $3 \mathbf{l}$ & 72 & $75: 25$ \\
\hline
\end{tabular}

With aliphatic epoxides the $\mathrm{S}_{\mathrm{N}} 2$-product 2 was formed exclusively in high yield as a diastereomeric mixture (entries 1-5). The same is true for glycidyl ethers (entries 6-8). In contrast, with aryl-substituted epoxides only the formation of the $\mathrm{S}_{\mathrm{N}} 1$-product 3 was observed, giving rise to $\beta$-substituted phenylalanines (entries 9-11). Obviously, under the mild reaction conditions the nucleophilic attack on the in situ formed benzylic carbenium ion is significantly faster than the $\mathrm{S}_{\mathrm{N}} 2$-attack.

To prove if this concept is also suitable for the modification of peptides, we subjected a phenylalanine dipeptide to analogous reaction conditions. Only the amount of base had to be increased to 3.5 equiv. LHMDS (lithium hexamethyldisilazide). To make the NMR spectra more clear by reducing the number of stereoisomers, the ring opening products were directly oxidized to the corresponding ketones 4 (Table 2). The yields obtained were slightly lower compared to the amino ester enolates but with $64-72 \%$ (over both steps) still in a preparative useful range.

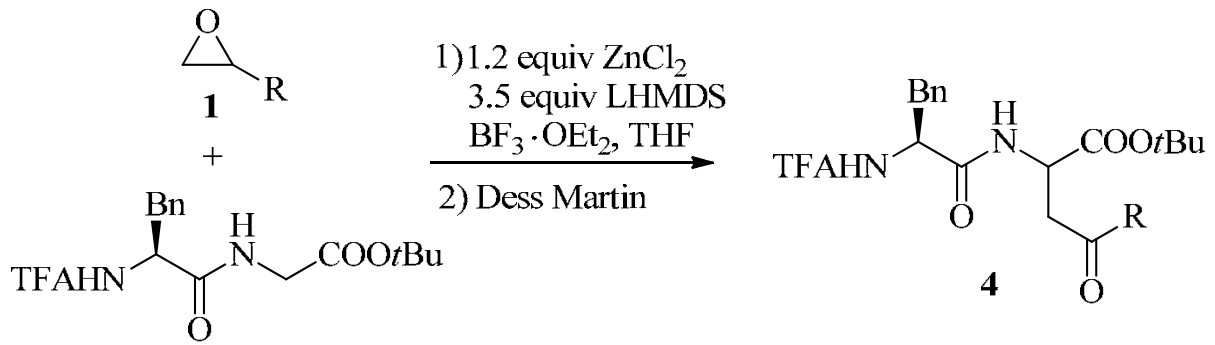

Table 2. Regioselective epoxide opening with chelated peptide enolates

\begin{tabular}{cccccc}
\hline Entry & Epoxide & $\mathrm{R}$ & Product & Yield (\%) & Diast.-ratio \\
\hline 1 & $\mathbf{1 a}$ & $\mathrm{CH}_{3}$ & $\mathbf{4 a}$ & 64 & $68: 32$ \\
2 & $\mathbf{1 b}$ & $\mathrm{C}_{2} \mathrm{H}_{5}$ & $\mathbf{4 b}$ & 68 & $67: 33$ \\
3 & $\mathbf{1 d}$ & $\mathrm{ClCH}_{2}$ & $\mathbf{4 d}$ & 66 & $68: 32$ \\
4 & $\mathbf{1 f}$ & $\mathrm{C}_{6} \mathrm{H}_{5} \mathrm{OCH}_{2}$ & $\mathbf{4 f}$ & 66 & $70: 30$ \\
5 & $\mathbf{1 h}$ & $p-\mathrm{CH}_{3} \mathrm{OC}_{6} \mathrm{H}_{4} \mathrm{OCH}_{2}$ & $\mathbf{4 h}$ & 65 & $69: 31$ \\
6 & $\mathbf{1 m}$ & $p-\mathrm{ClC}_{6} \mathrm{H}_{4} \mathrm{OCH}_{2}$ & $\mathbf{4 m}$ & 68 & $64: 36$ \\
7 & $\mathbf{1 n}$ & $p-\mathrm{NO}_{2} \mathrm{C}_{6} \mathrm{H}_{4} \mathrm{OCH}_{2}$ & $\mathbf{4 n}$ & 72 & $68: 32$ \\
\hline
\end{tabular}




\section{Conclusions}

In conclusion we could show that chelated enolates of $\alpha$-amino acids and peptides are suitable nucleophiles for the regioselective ring opening of epoxides. Depending on the substitution pattern, the reaction proceeds either in a $\mathrm{S}_{\mathrm{N}} 1$-type (arylepoxides) or a $\mathrm{S}_{\mathrm{N}} 2$-type (alkylepoxides) fashion. Attempts to increase the stereoselectivity of these processes as well as synthetic applications are currently under investigation.

\section{Experimental Section}

General. All reactions were carried out in oven-dried glassware $\left(100^{\circ} \mathrm{C}\right)$ under nitrogen. All solvents were dried before use: THF was distilled from $\mathrm{LiAlH}_{4}$. The products were purified by flash chromatography on silica gel $(0.063-0.2 \mathrm{~mm})$. Mixtures of ethyl acetate and hexanes were generally used as eluents. Analysis by TLC was carried out on commercially precoated Polygram SIL-G/UV 254 plates (Macherey-Nagel, Dueren). Visualization was accomplished with UV light, $\mathrm{KMnO}_{4}$ solution or iodine. ${ }^{1} \mathrm{H}$ - and ${ }^{13} \mathrm{C}$-NMR spectroscopic analysis was performed on a Bruker Avance II $400 \mathrm{MHz}$ spectrometer. Chemical shifts are reported on the $\delta$ (ppm) scale and the coupling constant are given in Hz. HRMS were measured with Finnigan MAT 95S mass spectrometer. Elemental analyses were carried out at the Department of Chemistry at Saarland University.

\section{General procedure for epoxide opening with amino acid enolates}

In a Schlenk tube hexamethyldisilazane $(497 \mathrm{mg}, 3.08 \mathrm{mmol})$ was dissolved in dry THF $(5 \mathrm{~mL})$ under Ar. After the solution was cooled to $-78{ }^{\circ} \mathrm{C}$, a $1.6 \mathrm{M}$ solution of $n$-BuLi $(1.72 \mathrm{~mL}, 2.75$ mmol) was added slowly. The solution was stirred for $10 \mathrm{~min}$ before the cooling bath was removed and the solution was stirred for a further $10 \mathrm{~min}$. In a second Schlenk flask $\mathrm{ZnCl}_{2}(180$ $\mathrm{mg}, 1.32 \mathrm{mmol})$ was dried with a heat gun under vacuum and dissolved in THF $(5 \mathrm{~mL})$ under Ar. Tfa-Gly-OtBu ( $250 \mathrm{mg}, 1.1 \mathrm{mmol})$ was added and the solution was cooled to $-78{ }^{\circ} \mathrm{C}$ before the LHMDS solution was added slowly via syringe. The resulting solution was stirred for $30 \mathrm{~min}$ at $-78{ }^{\circ} \mathrm{C}$. Then the corresponding epoxide $(1.65 \mathrm{mmol})$ was added followed by $\mathrm{BF}_{3} \mathrm{OEt}_{2}(78.1$ $\mathrm{mg}, 0.55 \mathrm{mmol}$ ) directly to the enolate at $-78{ }^{\circ} \mathrm{C}$. The reaction mixture was allowed to warm to r.t. over night before it was hydrolyzed with $1 \mathrm{M} \mathrm{HCl}$ and extracted thrice with ethyl acetate. The combined organic layers were dried $\left(\mathrm{Na}_{2} \mathrm{SO}_{4}\right)$, the solvent was evaporated in vacuo, and the crude product was purified by flash chromatography (silica gel, hexanes/EtOAc).

tert-Butyl 4-hydroxy-2-(2,2,2-trifluoroacetamido)pentanoate (2a). Major diastereomer (68\%): ${ }^{1} \mathrm{H}$ NMR $\left(400 \mathrm{MHz}, \mathrm{CDCl}_{3}\right): \delta=7.66(\mathrm{~d}, J=5.5 \mathrm{~Hz}, 1 \mathrm{H}), 4.63(\mathrm{td}, J=8.2,3.6 \mathrm{~Hz}, 1 \mathrm{H}), 3.85$ (m, $1 \mathrm{H}), 2.74$ (d, $J=3.2 \mathrm{~Hz}, 1 \mathrm{H}), 1.91$ (ddd, $J=14.2,10.4,3.7 \mathrm{~Hz}, 1 \mathrm{H}), 1.83$ (ddd, $J=11.1$, 8.5, $2.6 \mathrm{~Hz}, 1 \mathrm{H}), 1.48(\mathrm{~s}, 9 \mathrm{H}), 1.24(\mathrm{~d}, J=6.2 \mathrm{~Hz}, 3 \mathrm{H}) .{ }^{13} \mathrm{C} \mathrm{NMR}\left(100 \mathrm{MHz}, \mathrm{CDCl}_{3}\right): \delta=$ 169.7, $157.4(J=37.2 \mathrm{~Hz}), 115.7(J=285.6 \mathrm{~Hz}), 83.2,64.8,51.5,39.9,27.8$, 23.5. Minor 
diastereomer (32\%): ${ }^{1} \mathrm{H}$ NMR (400 MHz, $\left.\mathrm{CDCl}_{3}\right): \delta=7.53(\mathrm{~d}, J=4.0 \mathrm{~Hz}, 1 \mathrm{H}), 4.43(\mathrm{q}, J=6.2$ $\mathrm{Hz}, 1 \mathrm{H}), 3.97$ (m, $1 \mathrm{H}), 2.04$ (ddd, $J=14.5,5.7,3.2 \mathrm{~Hz}, 1 \mathrm{H}), 1.98$ (bs, $1 \mathrm{H}), 1.90$ (ddd, $J=$ 14.5, 9.4, 6.5 Hz, $1 \mathrm{H}), 1.47$ (s, $9 \mathrm{H}), 1.24$ (d, $J=6.0 \mathrm{~Hz}, 3 \mathrm{H}) .{ }^{13} \mathrm{C} \mathrm{NMR}\left(100 \mathrm{MHz}, \mathrm{CDCl}_{3}\right): \delta=$ 169.8, $156.8(J=37.3 \mathrm{~Hz}), 115.6(J=285.8 \mathrm{~Hz}), 83.1,65.5,52.1,39.4,27.8,24.1$. HRMS (CI) calcd. for $\mathrm{C}_{11} \mathrm{H}_{18} \mathrm{~F}_{3} \mathrm{NO}_{4}[\mathrm{M}+\mathrm{H}]^{+}:$286.1221. Found: 286.1273. Anal. Calcd for $\mathrm{C}_{11} \mathrm{H}_{18} \mathrm{~F}_{3} \mathrm{NO}_{4}$ (285.11): C 46.31; H 6.36; N 4.91. Found: C 46.46; H 6.21; N 5.18.

tert-Butyl 4-hydroxy-2-(2,2,2-trifluoroacetamido)hexanoate (2b). Major diastereomer (68\%): ${ }^{1} \mathrm{H}$ NMR (400 MHz, $\left.\mathrm{CDCl}_{3}\right): \delta=7.75(\mathrm{~d}, J=6.3 \mathrm{~Hz}, 1 \mathrm{H}), 4.66(\mathrm{~m}, 1 \mathrm{H}), 3.58(\mathrm{~m}, 1 \mathrm{H}), 2.68$ (bs, $1 \mathrm{H}), 1.87-1.93(\mathrm{~m}, 2 \mathrm{H}), 1.51-1.54(\mathrm{~m}, 2 \mathrm{H}), 1.49(\mathrm{~s}, 9 \mathrm{H}), 0.95(\mathrm{t}, J=7.5 \mathrm{~Hz}, 3 \mathrm{H}) .{ }^{13} \mathrm{C}$ NMR (100 MHz, $\left.\mathrm{CDCl}_{3}\right): \delta=169.7,157.4(\mathrm{~J}=37.2 \mathrm{~Hz}), 115.7(\mathrm{~J}=285.6 \mathrm{~Hz}), 83.2,70.1,52.4$, 38.2, 30.4, 27.9, 9.8. Minor diastereomer (32\%, selected signals): ${ }^{1} \mathrm{H}$ NMR (400 $\left.\mathrm{MHz}, \mathrm{CDCl}_{3}\right): \delta$ $=7.46(\mathrm{bs}, 1 \mathrm{H}), 4.42(\mathrm{~m}, 1 \mathrm{H}), 3.70(\mathrm{~m}, 1 \mathrm{H}), 2.11(\mathrm{ddd}, J=14.5,5.8,2.7 \mathrm{~Hz}, 1 \mathrm{H}) .{ }^{13} \mathrm{C}$ NMR $\left(100 \mathrm{MHz}, \mathrm{CDCl}_{3}\right): \delta=169.9,156.8,(J=37.3 \mathrm{~Hz}), 115.6(\mathrm{~J}=285.8 \mathrm{~Hz}), 83.1,70.6,52.1,37.1$, 30.7, 27.8, 9.5. HRMS (CI) calcd. for $\mathrm{C}_{12} \mathrm{H}_{20} \mathrm{~F}_{3} \mathrm{NO}_{4}[\mathrm{M}]^{+}:$299.1344. Found: 299.1329. Anal. Calcd for $\mathrm{C}_{12} \mathrm{H}_{20} \mathrm{~F}_{3} \mathrm{NO}_{4}$ (299.13): C 48.16; $\mathrm{H}$ 6.74; $\mathrm{N} 4.68$. Found: C 48.08; H 6.47; N 4.64.

tert-Butyl 4-hydroxy-2-(2,2,2-trifluoroacetamido)octanoate (2c). Major diastereomer (66\%): ${ }^{1} \mathrm{H}$ NMR (400 MHz, $\left.\mathrm{CDCl}_{3}\right): \delta=7.85(\mathrm{~d}, J=6.3 \mathrm{~Hz}, 1 \mathrm{H}), 4.63(\mathrm{~m}, 1 \mathrm{H}), 3.64(\mathrm{~m}, 1 \mathrm{H}), 2.83$ (bs, $1 \mathrm{H}), 1.85-1.88(\mathrm{~m}, 2 \mathrm{H}), 1.47(\mathrm{~s}, 9 \mathrm{H}), 1.23-1.37(\mathrm{~m}, 6 \mathrm{H}), 0.88(\mathrm{t}, J=6.9 \mathrm{~Hz}, 3 \mathrm{H}) .{ }^{13} \mathrm{C}$ NMR $\left(100 \mathrm{MHz}, \mathrm{CDCl}_{3}\right): \delta=169.7,157.4(\mathrm{~J}=37.2 \mathrm{~Hz}), 115.7(\mathrm{~J}=285.6 \mathrm{~Hz}), 83.1,68.8,51.5$, 37.7, 37.2, 27.8, 27.6, 22.4, 13.9. Minor diastereomer (34\%, selected signals): ${ }^{1} \mathrm{H}$ NMR (400 $\left.\mathrm{MHz}, \mathrm{CDCl}_{3}\right): \delta=7.56$ (bs, $\left.1 \mathrm{H}\right), 4.43(\mathrm{~m}, 1 \mathrm{H}), 3.70(\mathrm{~m}, 1 \mathrm{H}), 2.07$ (ddd, $J=14.5,5.8 \mathrm{~Hz}, 2.6$ $\mathrm{Hz}, 1 \mathrm{H}), 1.48(\mathrm{~s}, 9 \mathrm{H}) .{ }^{13} \mathrm{C} \mathrm{NMR}\left(100 \mathrm{MHz}, \mathrm{CDCl}_{3}\right): \delta=169.9,156.8,(\mathrm{~J}=37.3 \mathrm{~Hz}), 115.6(\mathrm{~J}=$ $285.8 \mathrm{~Hz}$ ), 83.0, 69.3, 52.2, 38.4, 37.7, 27.8, 27.4. HRMS (CI) calcd. for $\mathrm{C}_{14} \mathrm{H}_{24} \mathrm{~F}_{3} \mathrm{NO}_{4}$ [M$\left.\mathrm{C}_{4} \mathrm{H}_{9}\right]^{+}: 270.0953$. Found: 270.0942. Anal. Calcd for $\mathrm{C}_{14} \mathrm{H}_{24} \mathrm{~F}_{3} \mathrm{NO}_{4}(327.33): \mathrm{C} 51.37 ; \mathrm{H} 7.39 ; \mathrm{N}$ 4.28. Found: C 51.41; H 7.38; N 3.80.

tert-Butyl 5-chloro-4-hydroxy-2-(2,2,2-trifluoroacetamido)pentanoate (2d). Major diastereo mer (56\%): ${ }^{1} \mathrm{H}$ NMR $\left(400 \mathrm{MHz}, \mathrm{CDCl}_{3}\right): \delta=7.70(\mathrm{~d}, J=7.3 \mathrm{~Hz}, 1 \mathrm{H}), 4.68(\mathrm{dt}, J=7.7,3.8 \mathrm{~Hz}$, $1 \mathrm{H}), 3.87(\mathrm{~m}, 1 \mathrm{H}), 3.53(\mathrm{dd}, J=10.0,4.0 \mathrm{~Hz}, 1 \mathrm{H}), 3.49(\mathrm{dd}, J=10.0,5.2 \mathrm{~Hz}, 1 \mathrm{H}), 3.35(\mathrm{~d}, J=$ $3.9 \mathrm{~Hz}, 1 \mathrm{H}), 1.94-2.08(\mathrm{~m}, 2 \mathrm{H}), 1.47(\mathrm{~s}, 9 \mathrm{H}) .{ }^{13} \mathrm{C} \mathrm{NMR}\left(100 \mathrm{MHz}, \mathrm{CDCl}_{3}\right): \delta=169.2,157.4$ $(J=37.2 \mathrm{~Hz}), 115.7(J=285.6 \mathrm{~Hz}), 83.7,66.7,51.1,48.7,35.5,27.8$. Minor diastereomer $(44 \%$, selected signals): ${ }^{1} \mathrm{H} \mathrm{NMR}\left(400 \mathrm{MHz}, \mathrm{CDCl}_{3}\right): \delta=7.46(\mathrm{~d}, J=6.0 \mathrm{~Hz}, 1 \mathrm{H}), 4.50(\mathrm{q}, J=5.9 \mathrm{~Hz}$, $1 \mathrm{H}), 3.95$ (m, $1 \mathrm{H}), 3.57$ (dd, $J=11.2,4.1 \mathrm{~Hz}, 1 \mathrm{H}), 3.49(\mathrm{dd}, J=11.2,6.6 \mathrm{~Hz}, 1 \mathrm{H}), 2.72(\mathrm{~d}, J$ $=5.1 \mathrm{~Hz}, 1 \mathrm{H}), 2.20(\mathrm{ddd}, J=14.5,5.9,2.9 \mathrm{~Hz}, 1 \mathrm{H}), 2.02(\mathrm{~m}, 1 \mathrm{H}), 1.47(\mathrm{~s}, 9 \mathrm{H}) .{ }^{13} \mathrm{C} \mathrm{NMR}(100$ $\left.\mathrm{MHz}_{\mathrm{CDCl}}\right): \delta=169.4,157.4(\mathrm{~J}=37.2 \mathrm{~Hz}), 115.7(\mathrm{~J}=285.6 \mathrm{~Hz}), 83.6,68.4,51.3,49.3,35.0$, 27.7. HRMS (CI) calcd. for $\mathrm{C}_{11} \mathrm{H}_{17} \mathrm{ClF}_{3} \mathrm{NO}_{4}[\mathrm{M}+\mathrm{H}]^{+}:$321.0769. Found: 321.0795. Anal. Calcd for $\mathrm{C}_{11} \mathrm{H}_{17} \mathrm{ClF}_{3} \mathrm{NO}_{4}$ (319.07): C 41.32; $\mathrm{H}$ 5.36; $\mathrm{N}$ 4.38. Found: $\mathrm{C} 41.43 ; \mathrm{H}$ 5.08; N 4.54.

tert-Butyl 4-hydroxy-2-(2,2,2-trifluoroacetamido)non-8-enoate (2e). Major diastereomer (65\%): ${ }^{1} \mathrm{H}$ NMR (400 MHz, $\left.\mathrm{CDCl}_{3}\right): \delta=7.70(\mathrm{~d}, J=7.3 \mathrm{~Hz}, 1 \mathrm{H}), 5.78(\mathrm{~m}, 1 \mathrm{H}), 4.93-5.02(\mathrm{~m}$, $2 \mathrm{H}), 4.65$ (dt, $J=7.7,4.3 \mathrm{~Hz}, 1 \mathrm{H}), 3.62$ (m, $1 \mathrm{H}), 2.72$ (bs, $1 \mathrm{H}), 2.03-2.08$ (m, $2 \mathrm{H}), 1.81-1.92$ $(\mathrm{m}, 4 \mathrm{H}), 1.48(\mathrm{~s}, 9 \mathrm{H}), 1.25-1.42(\mathrm{~m}, 4 \mathrm{H}) .{ }^{13} \mathrm{C} \mathrm{NMR}\left(100 \mathrm{MHz}, \mathrm{CDCl}_{3}\right): \delta=169.8,136.6$, 
114.5, $157.4(J=37.2 \mathrm{~Hz}), 115.7(J=285.6 \mathrm{~Hz}), 83.3,68.6,51.4,38.8,37.3,33.5,28.6,27.9$, 24.9. Minor diastereomer (35\%, selected signals) ${ }^{1} \mathrm{H}$ NMR (400 MHz, $\left.\mathrm{CDCl}_{3}\right): \delta=7.51(\mathrm{bs}, 1 \mathrm{H})$, $5.77(\mathrm{~m}, 1 \mathrm{H}), 4.93-5.01(\mathrm{~m}, 2 \mathrm{H}), 4.64$ (q, $J=6.0 \mathrm{~Hz}, 1 \mathrm{H}), 3.75$ (bs, $1 \mathrm{H}), 2.02-2.10(\mathrm{~m}, 3 \mathrm{H})$, 1.85-1.92 (m, $2 \mathrm{H}), 1.47$ (s, $9 \mathrm{H}), 1.28-1.41(\mathrm{~m}, 4 \mathrm{H}) .{ }^{13} \mathrm{C} \mathrm{NMR}\left(100 \mathrm{MHz}, \mathrm{CDCl}_{3}\right): \delta=169.9$, $157.6(\mathrm{~J}=37.2 \mathrm{~Hz}), 138.5,115.7(\mathrm{~J}=285.6 \mathrm{~Hz}), 114.5,83.1,69.2,52.1,37.8,37.7,33.5,28.6$, 27.8, 24.7. HRMS (CI) calcd. for $\mathrm{C}_{16} \mathrm{H}_{26} \mathrm{~F}_{3} \mathrm{NO}_{4}[\mathrm{M}+1]^{+}: 354.1847$. Found: 357.1879. Anal. Calcd for $\mathrm{C}_{16} \mathrm{H}_{26} \mathrm{~F}_{3} \mathrm{NO}_{4}$ (353.37): C 54.38; H 7.42; N 3.96. Found: C 54.40; H 7.10; N 4.42.

tert-Butyl 5-phenoxy-4-hydroxy-2-(2,2,2-trifluoroacetamido)pentanoate (2f). Major diastereomer (56\%): ${ }^{1} \mathrm{H} \mathrm{NMR}\left(400 \mathrm{MHz}, \mathrm{CDCl}_{3}\right): \delta=7.77(\mathrm{~d}, J=7.8 \mathrm{~Hz}, 1 \mathrm{H}), 7.23-7.29$ (m, 2 H), $6.95(\mathrm{dd}, J=7.4 \mathrm{~Hz}, 1 \mathrm{H}), 6.85-6.87$ (m, $2 \mathrm{H}), 4.71(\mathrm{dt}, J=7.5,4.4 \mathrm{~Hz}, 1 \mathrm{H}), 4.06(\mathrm{~m}, 1 \mathrm{H})$, $3.91(\mathrm{dd}, J=7.9,3.1 \mathrm{~Hz}, 1 \mathrm{H}), 3.88(\mathrm{dd}, J=7.8,5.2 \mathrm{~Hz}, 1 \mathrm{H}), 3.83$ (d, $J=3.3 \mathrm{~Hz}, 1 \mathrm{H}), 1.98-$ 2.09 (m, $2 \mathrm{H}), 1.47$ (s, $9 \mathrm{H}) .{ }^{13} \mathrm{C} \mathrm{NMR}\left(100 \mathrm{MHz} \mathrm{CDCl}_{3}\right): \delta=169.2,158.1,157.4(\mathrm{~J}=37.4 \mathrm{~Hz})$, 129.5, 121.4, $115.7(\mathrm{~J}=285.6 \mathrm{~Hz}), 114.4,83.4,71.3,67.4,51.2,34.5,27.9$. Minor diastereomer (44\%, selected signals): ${ }^{1} \mathrm{H}$ NMR $\left(400 \mathrm{MHz}, \mathrm{CDCl}_{3}\right): \delta=7.43$ (bs, $\left.1 \mathrm{H}\right), 7.23-7.28$ (m, $2 \mathrm{H}$ ), 6.96 (dd, $J=7.4$ Hz, 1 H), 6.85-6.87 (m, 2 H), 4.51 (q, J = 5.9 Hz, 1 H), 4.12 (m, 1 H), 3.94 (dd, $J=9.4,3.5 \mathrm{~Hz}, 1 \mathrm{H}), 3.88(\mathrm{dd}, J=9.3,7.1 \mathrm{~Hz}, 1 \mathrm{H}), 2.52(\mathrm{~d}, J=4.3 \mathrm{~Hz}, 1 \mathrm{H}), 2.15(\mathrm{~m}, 1 \mathrm{H})$, $1.47(\mathrm{~s}, 9 \mathrm{H}) .{ }^{13} \mathrm{C} \mathrm{NMR}\left(100 \mathrm{MHz}, \mathrm{CDCl}_{3}\right): \delta=169.4,158.1,157.4(\mathrm{~J}=37.4 \mathrm{~Hz}), 129.6,121.4$, $115.7(J=285.6 \mathrm{~Hz}), 114.5,83.4,71.4,67.4,51.7,33.8,27.8$. HRMS (CI) calcd. for $\mathrm{C}_{17} \mathrm{H}_{22} \mathrm{~F}_{3} \mathrm{NO}_{5}[\mathrm{M}]^{+}: 377.1450$. Found: 377.1447 . Anal. Calcd for $\mathrm{C}_{17} \mathrm{H}_{22} \mathrm{~F}_{3} \mathrm{NO}_{5}$ (377.35): C 54.11; H 5.88; N 3.71. Found: C 54.64; H 5.62; N 3.95.

tert-Butyl 4-hydroxy-5-(4-tolyloxy)-2-(2,2,2-trifluoroacetamido)pentanoate (2g). Major diastereomer (55\%): ${ }^{1} \mathrm{H}$ NMR $\left(400 \mathrm{MHz}, \mathrm{CDCl}_{3}\right): \delta=7.81(\mathrm{~d}, J=7.5 \mathrm{~Hz}, 1 \mathrm{H}), 7.08(\mathrm{~d}, J=8.3$ $\mathrm{Hz}, 2 \mathrm{H}), 6.77-6.80$ (m, $2 \mathrm{H}), 4.73$ (dt, $J=7.4,4.2 \mathrm{~Hz}, 1 \mathrm{H}), 4.11(\mathrm{~m}, 1 \mathrm{H}), 3.81-3.97$ (m, $2 \mathrm{H})$, $2.29(\mathrm{~s}, 3 \mathrm{H}), 2.03-2.13(\mathrm{~m}, 2 \mathrm{H}), 1.49(\mathrm{~s}, 9 \mathrm{H}) .{ }^{13} \mathrm{C}$ NMR $\left(100 \mathrm{MHz}, \mathrm{CDCl}_{3}\right): \delta=169.2,157.4$ $(J=37.4 \mathrm{~Hz}), 156.0,130.0,129.0,115.7\left(\mathrm{q}, J_{8, \mathrm{~F}}=285.6 \mathrm{~Hz}\right), 114.3,83.4,71.6,67.5,51.2,34.5$, 27.9, 20.4. Minor diastereomer (45\%, selected signals): ${ }^{1} \mathrm{H}$ NMR (400 $\left.\mathrm{MHz}, \mathrm{CDCl}_{3}\right): \delta=7.47$ $(\mathrm{d}, J=5.5 \mathrm{~Hz}, 1 \mathrm{H}), 4.53(\mathrm{q}, J=5.9 \mathrm{~Hz}, 1 \mathrm{H}), 2.28(\mathrm{~s}, 3 \mathrm{H}), 2.03-2.13(\mathrm{~m}, 2 \mathrm{H}), 1.49(\mathrm{~s}, 9 \mathrm{H})$. ${ }^{13} \mathrm{C} \mathrm{NMR}\left(100 \mathrm{MHz}, \mathrm{CDCl}_{3}\right): \delta=169.2,155.8,130.7,83.3,71.6,67.4,51.6,33.8,27.8$. HRMS (CI) calcd. for $\mathrm{C}_{18} \mathrm{H}_{24} \mathrm{~F}_{3} \mathrm{NO}_{5}[\mathrm{M}]^{+}: 391.1607$. Found: 391.1616 .

tert-Butyl 4-hydroxy-5-(4-methoxyphenoxy)-2-(2,2,2-trifluoroacetamido)pentanoate (2h). Major diastereomer (60\%): ${ }^{1} \mathrm{H}$ NMR $\left(400 \mathrm{MHz}, \mathrm{CDCl}_{3}\right): \delta=7.78(\mathrm{~d}, J=7.0 \mathrm{~Hz}, 1 \mathrm{H}), 6.83-6.85$ $(\mathrm{m}, 4 \mathrm{H}), 4.73$ (dt, $J=7.4,4.5 \mathrm{~Hz}, 1 \mathrm{H}), 4.10(\mathrm{~m}, 1 \mathrm{H}), 3.79-3.93(\mathrm{~m}, 2 \mathrm{H}), 3.77$ (s, $3 \mathrm{H}), 3.11$ (d, $J=3.3 \mathrm{~Hz}, 1 \mathrm{H}), 2.03-2.13(\mathrm{~m}, 2 \mathrm{H}), 1.49(\mathrm{~s}, 9 \mathrm{H}) .{ }^{13} \mathrm{C} \mathrm{NMR}\left(100 \mathrm{MHz}, \mathrm{CDCl}_{3}\right): \delta=171.5$, $157.4(J=37.4 \mathrm{~Hz}), 152.2,130.0,129.0,115.7(\mathrm{~J}=285.6 \mathrm{~Hz}), 115.4,83.4,72.6,65.5,55.8$, 51.2, 36.5, 27.9. Minor diastereomer (40\%, selected signals): ${ }^{1} \mathrm{H}$ NMR (400 $\left.\mathrm{MHz}, \mathrm{CDCl}_{3}\right): \delta=$ 7.44 (s, $1 \mathrm{H}), 4.53$ (q, J = 5.9 Hz, $1 \mathrm{H}), 4.10$ (m, $1 \mathrm{H}), 3.77$ (s, $3 \mathrm{H}), 2.19-2.25$ (m, $2 \mathrm{H}), 1.49$ (s, $9 \mathrm{H}) .{ }^{13} \mathrm{C} \mathrm{NMR}\left(100 \mathrm{MHz}, \mathrm{CDCl}_{3}\right): \delta=171.3,152.1,129.9,129.0,115.5,83.4,72.6,65.5,55.6$, 51.2, 36.9, 27.8. HRMS (CI) calcd. for $\mathrm{C}_{18} \mathrm{H}_{24} \mathrm{~F}_{3} \mathrm{NO}_{6}[\mathrm{M}]^{+}: 407.1556$. Found: 407.1550.

tert-Butyl 4-hydroxy-3-phenyl-2-(2,2,2-trifluoroacetamido)butanoate (3i). Major diastereomer (75\%): ${ }^{1} \mathrm{H}$ NMR (400 $\left.\mathrm{MHz} \mathrm{CDCl}_{3}\right): \delta=7.66(\mathrm{~d}, J=7.5 \mathrm{~Hz}, 1 \mathrm{H}), 7.22-7.31(\mathrm{~m}, 5$ 
H), 4.79 (dd, $J=7.7 \mathrm{~Hz}, 1 \mathrm{H}), 3.91-3.99(\mathrm{~m}, 2 \mathrm{H}), 3.19(\mathrm{dt}, J=7.6,5.3 \mathrm{~Hz}, 1 \mathrm{H}), 2.66(\mathrm{~s}, 1 \mathrm{H})$, $1.24(\mathrm{~s}, 9 \mathrm{H}) .{ }^{13} \mathrm{C} \mathrm{NMR}\left(100 \mathrm{MHz}, \mathrm{CDCl}_{3}\right): \delta=168.9,157.1$ (q, $\left.J=37.4 \mathrm{~Hz}\right), 137.1,128.6$, 128.5, 127.8, 115.6 (q, $J=285.8 \mathrm{~Hz}), 83.3,63.7,55.8,49.3,27.5$. Minor diastereomer (25\%, selected signals): ${ }^{1} \mathrm{H}$ NMR (400 MHz, $\left.\mathrm{CDCl}_{3}\right): \delta=7.29-7.33(\mathrm{~m}, 3 \mathrm{H}), 7.07-7.06(\mathrm{~m}, 2 \mathrm{H}), 7.03$ $(\mathrm{d}, J=7.2 \mathrm{~Hz}, 1 \mathrm{H}), 5.03(\mathrm{dd}, J=7.9,3.4 \mathrm{~Hz}, 1 \mathrm{H}), 3.73-3.83(\mathrm{~m}, 2 \mathrm{H}), 3.56$ (ddd, 9.6, 5.6, 3.4 $\mathrm{Hz}, 1 \mathrm{H}), 3.39(\mathrm{dd}, J=8.1,6.2 \mathrm{~Hz}, 1 \mathrm{H}), 1.41(\mathrm{~s}, 9 \mathrm{H}) .{ }^{13} \mathrm{C} \mathrm{NMR}\left(100 \mathrm{MHz}, \mathrm{CDCl}_{3}\right): \delta=168.5$, 135.3, 128.8, 128.4, 128.3, $115.6(\mathrm{~J}=285.5 \mathrm{~Hz}), 84.1,62.1,53.5,50.3,27.9$ HRMS (CI) calcd. for $\mathrm{C}_{12} \mathrm{H}_{11} \mathrm{~F}_{3} \mathrm{NO}_{4}\left[\mathrm{M}-\mathrm{C}_{4} \mathrm{H}_{9}\right]^{+}:$290.0646. Found: 290.0612. Anal. Calcd for for $\mathrm{C}_{16} \mathrm{H}_{20} \mathrm{~F}_{3} \mathrm{NO}_{4}$ (347.13): C 55.33; H 5.80; N 4.03. Found: C 54.99; H 5.81; N 4.29.

tert-Butyl 4-hydroxy-3-(4-methoxyphenyl)-2-(2,2,2-trifluoroacetamido)butanoate (3k). Major diastereomer (67\%): ${ }^{1} \mathrm{H}$ NMR $\left(400 \mathrm{MHz}, \mathrm{CDCl}_{3}\right): \delta=7.48(\mathrm{~d}, J=8.1 \mathrm{~Hz}, 1 \mathrm{H}), 6.85-6.95$ $(\mathrm{m}, 4 \mathrm{H}), 4.90(\mathrm{t}, J=6.8 \mathrm{~Hz}, 1 \mathrm{H}), 4.10(\mathrm{dd}, J=11.2,6.4 \mathrm{~Hz}, 1 \mathrm{H}), 4.00(\mathrm{dd}, J=11.1,5.4 \mathrm{~Hz}, 1$ $\mathrm{H}), 3.83(\mathrm{~s}, 3 \mathrm{H}), 3.19(\mathrm{dt}, J=7.6,5.3 \mathrm{~Hz}, 1 \mathrm{H}), 1.32(\mathrm{~s}, 9 \mathrm{H}) .{ }^{13} \mathrm{C} \mathrm{NMR}\left(100 \mathrm{MHz}, \mathrm{CDCl}_{3}\right): \delta=$ 168.7, $157.2(J=37.4 \mathrm{~Hz}), 156.9,135.6,121.1,115.6(\mathrm{~J}=285.8 \mathrm{~Hz}), 114.6,82.8,63.2,55.3$, 55.2, 43.0, 27.7. Minor diastereomer (33\%, selected signals): ${ }^{1} \mathrm{H}$ NMR (400 $\left.\mathrm{MHz}, \mathrm{CDCl}_{3}\right): \delta=$ $7.74(\mathrm{~d}, J=5.9 \mathrm{~Hz}, 1 \mathrm{H}), 7.23-7.29(\mathrm{~m}, 4 \mathrm{H}), 4.95(\mathrm{dd}, J=8.2,5.0 \mathrm{~Hz}, 1 \mathrm{H}), 4.00$ (dd, $J=11.1$, $5.4 \mathrm{~Hz}, 1 \mathrm{H}), 3.84(\mathrm{~s}, 3 \mathrm{H}), 1.28(\mathrm{~s}, 9 \mathrm{H}) .{ }^{13} \mathrm{C} \mathrm{NMR}\left(100 \mathrm{MHz}, \mathrm{CDCl}_{3}\right): \delta=168.7,156.5,136.1$, 114.9, 83.2, 63.2, 55.2, 45.5, 27.7. HRMS (CI) calcd. for $\mathrm{C}_{17} \mathrm{H}_{22} \mathrm{~F}_{3} \mathrm{NO}_{5}[\mathrm{M}+1]^{+}: 378.1484$. Found: 378.1499.

tert-Butyl 4-hydroxy-3-(2-chlorophenyl)-2-(2,2,2-trifluoroacetamido)butanoate (3l). Major diastereomer (75\%): ${ }^{1} \mathrm{H}$ NMR $\left(400 \mathrm{MHz}, \mathrm{CDCl}_{3}\right): \delta=7.46(\mathrm{~d}, J=7.5 \mathrm{~Hz}, 1 \mathrm{H}), 7.30-7.32(\mathrm{~m}, 2$ H), 7.23-7.26 (m, $2 \mathrm{H}), 4.83(\mathrm{t}, J=7.8 \mathrm{~Hz}, 1 \mathrm{H}), 3.98(\mathrm{dd}, J=4.8,1.9 \mathrm{~Hz}, 1 \mathrm{H}), 3.94(\mathrm{dd}, J=$ 4.8, $1.9 \mathrm{~Hz}), 3.19(\mathrm{dt}, J=7.6,4.8 \mathrm{~Hz}, 1 \mathrm{H}), 1.30(\mathrm{~s}, 9 \mathrm{H}) \cdot{ }^{13} \mathrm{C} \mathrm{NMR}\left(100 \mathrm{MHz}, \mathrm{CDCl}_{3}\right): \delta=$ $168.7,157.4(\mathrm{~J}=37.4 \mathrm{~Hz}), 135.9,133.8,129.9,129.7,127.5,126.2,115.6(\mathrm{~J}=285.8 \mathrm{~Hz}), 83.7$, 63.4, 55.5, 48.9, 27.6. Minor diastereomer (25\%, selected signals): $\left.{ }^{1} \mathrm{H} \mathrm{NMR} \mathrm{(400} \mathrm{MHz}, \mathrm{CDCl}_{3}\right)$ : $\delta=7.78(\mathrm{~d}, J=7.8 \mathrm{~Hz}, 1 \mathrm{H}), 5.02(\mathrm{dd}, J=7.8,3.4 \mathrm{~Hz}, 1 \mathrm{H}), 3.71-3.76(\mathrm{~m}, 2 \mathrm{H}), 3.55(\mathrm{ddd}, J=$ 9.6, 5.6, $3.4 \mathrm{~Hz}, 1 \mathrm{H}), 3.45$ (bs, $1 \mathrm{H}), 1.45$ (s, $9 \mathrm{H}) .{ }^{13} \mathrm{C} \mathrm{NMR}\left(100 \mathrm{MHz}, \mathrm{CDCl}_{3}\right): \delta=168.4$, 129.6, 129.0, 84.4, 62.0, 53.4, 49.7, 28.0. HRMS (CI) calcd. for $\mathrm{C}_{16} \mathrm{H}_{19} \mathrm{ClF}_{3} \mathrm{NO}_{4}[\mathrm{M}+1]^{+}$: 383.0925. Found: 383.0943 .

\section{General procedure for epoxide opening with dipeptide enolates}

In a oven-dried Schlenk tube hexamethyldisilazane (411 $\mathrm{mg}, 2.54 \mathrm{mmol})$ was dissolved in dry THF $(5.0 \mathrm{~mL})$ under Ar. After the solution was cooled to $-78{ }^{\circ} \mathrm{C}$, a $1.6 \mathrm{M}$ solution of $n$-BuLi $(1.5 \mathrm{~mL}, 2.34 \mathrm{mmol})$ was added slowly. The solution was stirred for $10 \mathrm{~min}$ before the cooling bath was removed and the solution was stirred for a further $10 \mathrm{~min}$. In a second Schlenk flask $\mathrm{ZnCl}_{2}$ (109 mg, $\left.0.80 \mathrm{mmol}\right)$ was dried with a heat gun under vacuum and dissolved in THF (2.0 $\mathrm{mL}$ ) under Ar. The corresponding dipeptide $(250 \mathrm{mg}, 0.67 \mathrm{mmol})$ was added and the solution was cooled to $-78{ }^{\circ} \mathrm{C}$ before the LHMDS solution was added slowly via syringe. The resulting solution was stirred for $60 \mathrm{~min}$ at $-78^{\circ} \mathrm{C}$. Then the corresponding epoxide $(1.0 \mathrm{mmol})$ was added directly to the enolate at $-78{ }^{\circ} \mathrm{C}$, followed by $\mathrm{BF}_{3} \cdot \mathrm{OEt}_{2}(47.4 \mathrm{mg}, 0.34 \mathrm{mmol})$. The reaction 
mixture was allowed to warm to r.t. over night before it was hydrolyzed with $1 \mathrm{M} \mathrm{HCl}$ and extracted three times with ethyl acetate. The combined organic layers were dried $\left(\mathrm{Na}_{2} \mathrm{SO}_{4}\right)$, the solvent was evaporated in vacuo, and the crude product was purified by flash chromatography (silica gel, hexanes/EtOAc).

\section{Oxidation of substituted peptides}

The combined organic layers of the epoxide opening reaction were dried $\left(\mathrm{Na}_{2} \mathrm{SO}_{4}\right)$ and the solvent was evaporated in vacuo. The crude product was re-dissolved in $10 \mathrm{ml}$ dry dichloromethane and Dess-Martin periodinane $(1.0 \mathrm{mmol})$ was added in one portion at $0{ }^{\circ} \mathrm{C}$. After the oxidation was complete (tlc), the reaction was quenched by a saturated aqueous solution of $\mathrm{NaHCO}_{3}$ containing $\mathrm{Na}_{2} \mathrm{~S}_{2} \mathrm{O}_{3}$ and the product was extracted three times with ethyl acetate. The combined organic layers were dried $\left(\mathrm{Na}_{2} \mathrm{SO}_{4}\right)$, the solvent was removed in vacuo, and the crude product was purified by flash chromatography (silica gel, hexanes/EtOAc).

tert-Butyl 4-oxo-2-((S)-3-phenyl-2-(2,2,2-trifluoroacetamido)propanamido)pentanoate (4a). Major diastereomer (68\%): ${ }^{1} \mathrm{H}$ NMR (400 MHz, $\left.\mathrm{CDCl}_{3}\right): \delta=7.23-7.34(\mathrm{~m}, 4 \mathrm{H}), 7.18(\mathrm{~m}, 1 \mathrm{H})$, $7.13(\mathrm{~d}, J=7.8 \mathrm{~Hz}, 1 \mathrm{H}), 6.68(\mathrm{~d}, J=7.5 \mathrm{~Hz}, 1 \mathrm{H}), 4.65(\mathrm{~m}, 1 \mathrm{H}), 4.60(\mathrm{td}, J=8.2,4.2 \mathrm{~Hz}, 1 \mathrm{H})$, $3.21(\mathrm{dd}, J=13.9,6.4 \mathrm{~Hz}, 1 \mathrm{H}), 3.07-3.17$ (m, $2 \mathrm{H}), 2.90$ (dd, $J=18.3,9.0 \mathrm{~Hz}, 1 \mathrm{H}), 2.14$ (s, 3 $\mathrm{H}), 1.44(\mathrm{~s}, 9 \mathrm{H}) .{ }^{13} \mathrm{C} \mathrm{NMR}\left(100 \mathrm{MHz}, \mathrm{CDCl}_{3}\right): \delta=206.3,168.9,168.6,156.9(\mathrm{~J}=37.5 \mathrm{~Hz})$, 134.8, 129.3, 128.7, 127.4, $115.6(J=285.6 \mathrm{~Hz}), 82.8,54.2$, 48.9, 44.7, 38.3, 29.8, 27.8. Minor diastereomer (32\%, selected signals): ${ }^{1} \mathrm{H}$ NMR (400 MHz, $\left.\mathrm{CDCl}_{3}\right): \delta=7.57(\mathrm{bs}, 1 \mathrm{H}), 7.45(\mathrm{bs}, 1$ H), 7.14-7.27 (m, 5 H), 4.74 (bs, 2 H), 3.20 (dd, $J=13.9,6.1 \mathrm{~Hz}, 1 \mathrm{H}), 2.91$ (dd, $J=18.3,4.2$ $\mathrm{Hz}, 1 \mathrm{H}), 2.12$ (s, $3 \mathrm{H}), 1.37$ (s, $9 \mathrm{H}) .{ }^{13} \mathrm{C} \mathrm{NMR}\left(100 \mathrm{MHz}, \mathrm{CDCl}_{3}\right): \delta=206.2,168.7,168.4$, 134.8, 129.3, 128.7, 127.4, 82.8, 54.2, 48.9, 44.5, 38.3, 29.8, 27.7. HRMS (CI) calcd. for $\mathrm{C}_{20} \mathrm{H}_{25} \mathrm{~F}_{3} \mathrm{~N}_{2} \mathrm{O}_{5}[\mathrm{M}+1]^{+}: 431.1749$. Found: 431.1794 .

tert-Butyl 4-oxo-2-((S)-3-phenyl-2-(2,2,2-trifluoroacetamido)propanamido)hexanoate (4b). Major diastereomer (67\%): ${ }^{1} \mathrm{H}$ NMR $\left(400 \mathrm{MHz} \mathrm{CDCl}_{3}\right): \delta=7.16-7.31(\mathrm{~m}, 5 \mathrm{H}), 7.14(\mathrm{bs}, 1 \mathrm{H})$, $6.82(\mathrm{~d}, J=7.5 \mathrm{~Hz}, 1 \mathrm{H}), 4.69-4.74(\mathrm{~m}, 1 \mathrm{H}), 4.60$ (td, $J=7.8,4.3 \mathrm{~Hz}, 1 \mathrm{H}), 3.06-3.29(\mathrm{~m}, 4 \mathrm{H})$, 2.41-2.47 (m, $2 \mathrm{H}), 1.44(\mathrm{~s}, 9 \mathrm{H}), 1.05(\mathrm{t}, J=7.34 \mathrm{~Hz}, 3 \mathrm{H}) .{ }^{13} \mathrm{C}$ NMR $\left(100 \mathrm{MHz}, \mathrm{CDCl}_{3}\right): \delta=$ 200.7, 169.1, 168.6, $156.9(\mathrm{~J}=37.5 \mathrm{~Hz}), 134.9,129.2,128.7,127.4,115.6(\mathrm{~J}=285.6 \mathrm{~Hz}), 83.2$, 54.4, 48.9, 41.3, 35.6, 38.2, 27.6, 7.9. Minor diastereomer (33\%, selected signals): ${ }^{1} \mathrm{H}$ NMR (400 $\left.\mathrm{MHz}, \mathrm{CDCl}_{3}\right): \delta=7.14-7.31(\mathrm{~m}, 6 \mathrm{H}), 6.82(\mathrm{~d}, J=7.6 \mathrm{~Hz}, 1 \mathrm{H}), 4.71(\mathrm{~m}, 1 \mathrm{H}), 4.60(\mathrm{td}, J=7.9$, $4.3 \mathrm{~Hz}, 1 \mathrm{H}), 3.06-3.29(\mathrm{~m}, 4 \mathrm{H}), 2.41-2.47(\mathrm{~m}, 2 \mathrm{H}), 1.44(\mathrm{~s}, 9 \mathrm{H}), 1.06(\mathrm{t}, J=7.3 \mathrm{~Hz}, 3 \mathrm{H}) .{ }^{13} \mathrm{C}$ NMR (100 MHz, $\left.\mathrm{CDCl}_{3}\right): \delta=200.7,169.1,168.6,134.9,129.2,128.7,127.4,83.2,54.4,47.6$, 41.3, 38.2, 27.7, 8.0. HRMS (CI) calcd. for $\mathrm{C}_{21} \mathrm{H}_{27} \mathrm{~F}_{3} \mathrm{~N}_{2} \mathrm{O}_{5}[\mathrm{M}+1]^{+}: 445.1908$. Found: 445.1906.

tert-Butyl 5-chloro-4-oxo-2-((S)-3-phenyl-2-(2,2,2-trifluoroacetamido)propanamido) pentanoate (4d). Major diastereomer (68\%): ${ }^{1} \mathrm{H}$ NMR (400 MHz, $\left.\mathrm{CDCl}_{3}\right): \delta=7.16-7.31$ (m, 6 H), $6.82(\mathrm{~d}, J=7.5 \mathrm{~Hz}, 1 \mathrm{H}), 4.72(\mathrm{~m}, 1 \mathrm{H}), 4.60(\mathrm{td}, J=7.8,4.3 \mathrm{~Hz}, 1 \mathrm{H}), 4.07$ (d, $J=15.5 \mathrm{~Hz}$, $1 \mathrm{H}), 4.02(\mathrm{~d}, J=15.4 \mathrm{~Hz}, 1 \mathrm{H}), 3.06-3.29(\mathrm{~m}, 4 \mathrm{H}), 1.44(\mathrm{~s}, 9 \mathrm{H}) .{ }^{13} \mathrm{C} \mathrm{NMR}\left(100 \mathrm{MHz}, \mathrm{CDCl}_{3}\right)$ : $\delta=200.7,169.1,168.6,156.9(J=37.5 \mathrm{~Hz}), 134.9,129.2,128.7,127.4,115.6(J=285.6 \mathrm{~Hz})$, 
83.2, 54.4, 48.9, 47.6, 41.3, 38.2, 27.7. Minor diastereomer (32\%, selected signals): ${ }^{1} \mathrm{H}$ NMR $\left(400 \mathrm{MHz}, \mathrm{CDCl}_{3}\right): 6.81(\mathrm{~d}, J=7.5 \mathrm{~Hz}, 1 \mathrm{H}), 4.69-4.74(\mathrm{~m}, 2 \mathrm{H}), 4.07(\mathrm{~d}, J=15.5 \mathrm{~Hz}, 1 \mathrm{H})$, $4.02(\mathrm{~d}, J=15.4 \mathrm{~Hz}, 1 \mathrm{H}), 3.06-3.29(\mathrm{~m}, 4 \mathrm{H}), 1.45(\mathrm{~s}, 9 \mathrm{H}) .{ }^{13} \mathrm{C} \mathrm{NMR}\left(100 \mathrm{MHz}, \mathrm{CDCl}_{3}\right): \delta=$ 200.7, 169.1, 168.6, 129.2, 128.7, 127.4, 115, 83.2, 54.4, 48.9, 47.6, 41.3, 38.2, 27.7. HRMS (CI) calcd. for $\mathrm{C}_{20} \mathrm{H}_{24} \mathrm{ClF}_{3} \mathrm{~N}_{2} \mathrm{O}_{5}[\mathrm{M}]^{+}$: 464.1326. Found: 464.1326 .

tert-Butyl 5-phenoxy-4-oxo-2-((S)-3-phenyl-2-(2,2,2-trifluoroacetamido)propanamido) pentanoate (4f). Major diastereomer (70\%): ${ }^{1} \mathrm{H}$ NMR $\left(400 \mathrm{MHz}, \mathrm{CDCl}_{3}\right): \delta=7.13-7.33(\mathrm{~m}, 9$ $\mathrm{H}), 7.01(\mathrm{~m}, 1 \mathrm{H}), 6.85-6.88(\mathrm{~m}, 2 \mathrm{H}), 6.44(\mathrm{~d}, J=8.4 \mathrm{~Hz}, 1 \mathrm{H}), 4.63-4.70(\mathrm{~m}, 2 \mathrm{H}), 4.41(\mathrm{~s}, 2$ H), 3.11-3.20 (m, $2 \mathrm{H}), 2.98$ (dd, $J=13.5,8.8 \mathrm{~Hz}, 1 \mathrm{H}), 2.80$ (dd, $J=18.8,4.3 \mathrm{~Hz}, 1 \mathrm{H}), 1.36$ (s, $9 \mathrm{H}) .{ }^{13} \mathrm{C} \mathrm{NMR}\left(100 \mathrm{MHz}, \mathrm{CDCl}_{3}\right)$ (from mixture): $\delta=205.7,168.8,168.7,157.4,156.5(\mathrm{~J}=$ $37.3 \mathrm{~Hz}), 135.3,129.7,129.2,128.7,127.2,121.9,114.4,115.6(\mathrm{~J}=285.9 \mathrm{~Hz}), 82.9,72.3,54.6$, 48.1, 41.0, 38.5, 27.7. Minor diastereomer (30\%, selected signals): $\left.{ }^{1} \mathrm{H} \mathrm{NMR} \mathrm{(400} \mathrm{MHz}, \mathrm{CDCl}_{3}\right)$ : $\delta=6.58(\mathrm{~d}, J=7.6 \mathrm{~Hz}, 1 \mathrm{H}), 4.66-4.71(\mathrm{~m}, 1 \mathrm{H}), 4.62(\mathrm{q}, J=6.7 \mathrm{~Hz}, 1 \mathrm{H}), 4.53(\mathrm{~d}, J=6.8 \mathrm{~Hz}, 2$ $\mathrm{H}), 3.28(\mathrm{dd}, J=18.6,4.4 \mathrm{~Hz}, 1 \mathrm{H}), 1.44(\mathrm{~s}, 9 \mathrm{H}) .{ }^{13} \mathrm{C} \mathrm{NMR}\left(100 \mathrm{MHz}, \mathrm{CDCl}_{3}\right): \delta=205.9$, 169.0, 168.9, 134.9, 129.6, 129.2, 128.6, 127.3, 121.8, 115.5 ( $J=285.5 \mathrm{~Hz}), 82.9$, 72.4, 54.3, 48.6, 41.0, 38.2, 27.7. HRMS (CI) calcd. for $\mathrm{C}_{26} \mathrm{H}_{29} \mathrm{~F}_{3} \mathrm{~N}_{2} \mathrm{O}_{6}[\mathrm{M}]^{+}: 522.1978$. Found: 522.1953.

tert-Butyl 5-(4-methoxyphenoxy)-4-oxo-2-((S)-3-phenyl-2-(2,2,2-trifluoroacetamido) propanamido)pentanoate (4h). Major diastereomer (69\%): ${ }^{1} \mathrm{H} \mathrm{NMR}\left(400 \mathrm{MHz}, \mathrm{CDCl}_{3}\right): \delta=$ 7.17-7.31 (m, $5 \mathrm{H}), 7.01(\mathrm{~d}, J=7.3 \mathrm{~Hz}, 1 \mathrm{H}), 6.79-6.84(\mathrm{~m}, 4 \mathrm{H}), 6.53$ (d, J = 7.8 Hz, $1 \mathrm{H}), 4.68$ $(\mathrm{m}, 1 \mathrm{H}), 4.61(\mathrm{td}, J=13.4,6.6 \mathrm{~Hz}, 1 \mathrm{H}), 4.49(\mathrm{~d}, J=16.8 \mathrm{~Hz}, 1 \mathrm{H}), 3.76(\mathrm{~s}, 3 \mathrm{H}), 3.27$ (dd, $J=$ 18.5, 4.3 Hz, $1 \mathrm{H}), 3.18$ (dd, $J=13.9,6.7 \mathrm{~Hz}, 1 \mathrm{H}), 3.07-3.14(\mathrm{~m}, 2 \mathrm{H}), 1.44(\mathrm{~s}, 9 \mathrm{H}) .{ }^{13} \mathrm{C} \mathrm{NMR}$ $\left(100 \mathrm{MHz} \mathrm{CDCl}_{3}\right): \delta=205.9,168.8,168.4,156.9(\mathrm{~J}=37.5 \mathrm{~Hz}), 154.7,151.6,135.2,129.2$, 128.8, 127.4, $115.6(\mathrm{~J}=285.6 \mathrm{~Hz}), 115.5,114.9$, 83.0, 73.2, 55.7, 54.7, 48.2, 40.9, 38.6, 27.7. Minor diastereomer (31\%, selected signals): ${ }^{1} \mathrm{H} \mathrm{NMR}\left(400 \mathrm{MHz}, \mathrm{CDCl}_{3}\right): 7.14-7.30(\mathrm{~m}, 6 \mathrm{H})$, 6.81-6.87 (m, $4 \mathrm{H}), 6.31$ (d, J=7.8 Hz, 1 H), 4.60-4.69 (m, $2 \mathrm{H}), 4.37$ (s, $2 \mathrm{H}), 3.77$ (s, $3 \mathrm{H})$, 3.13-3.21 (m, $2 \mathrm{H}), 2.97$ (dd, $J=13.8,8.8 \mathrm{~Hz}, 1 \mathrm{H}), 2.79$ (dd, $J=18.7,4.3 \mathrm{~Hz}, 1 \mathrm{H}), 1.38$ (s, 9 H). ${ }^{13} \mathrm{C} \mathrm{NMR}\left(100 \mathrm{MHz}, \mathrm{CDCl}_{3}\right): \delta=206.2,168.8,168.6,156.9(\mathrm{~J}=37.5 \mathrm{~Hz}), 154.6,129.3$, 128.8, 127.5, $115.6(\mathrm{~J}=285.6 \mathrm{~Hz}), 115.5,115.0,114.8,83.1$, 73.3, 55.6, 54.3, 48.6, 41.0, 38.2, 27.8. HRMS (CI) calcd. for $\mathrm{C}_{27} \mathrm{H}_{31} \mathrm{~F}_{3} \mathrm{~N}_{2} \mathrm{O}_{7}[\mathrm{M}]^{+}: 552.2083$. Found: 552.2076.

tert-Butyl 5-(4-chlorophenoxy)-4-oxo-2-((S)-3-phenyl-2-(2,2,2-trifluoroacetamido)propanamido) pentanoate (4m). Major diastereomer (64\%): ${ }^{1} \mathrm{H}$ NMR (400 MHz, $\left.\mathrm{CDCl}_{3}\right): \delta=7.19-7.31$ (m, 5 H), $7.00(\mathrm{~d}, J=7.3 \mathrm{~Hz}, 1 \mathrm{H}), 6.76-6.83(\mathrm{~m}, 4 \mathrm{H}), 6.53$ (d, $J=7.8 \mathrm{~Hz}, 1 \mathrm{H}), 4.65-4.71(\mathrm{~m}, 1 \mathrm{H})$, $4.61(\mathrm{td}, J=13.4,6.6 \mathrm{~Hz}, 1 \mathrm{H}), 4.49$ (d, $J=16.8 \mathrm{~Hz}, 1 \mathrm{H}), 3.27(\mathrm{dd}, J=18.5,4.3 \mathrm{~Hz}, 1 \mathrm{H}), 3.18$ $(\mathrm{dd}, J=13.9,6.7 \mathrm{~Hz}, 1 \mathrm{H}), 3.07-3.14(\mathrm{~m}, 2 \mathrm{H}), 1.44(\mathrm{~s}, 9 \mathrm{H}) .{ }^{13} \mathrm{C} \mathrm{NMR}\left(100 \mathrm{MHz}, \mathrm{CDCl}_{3}\right): \delta=$ 205.9, 168.8, 168.4, $156.9(\mathrm{~J}=37.5 \mathrm{~Hz}), 154.7,151.6,135.2,129.3,128.8,127.3,115.6(\mathrm{~J}=$ $285.6 \mathrm{~Hz}), 115.4,83.0,73.2,55.6,54.7,48.1,40.9$, 38.6, 27.7. Minor diastereomer (36\%, selected signals): ${ }^{1} \mathrm{H}$ NMR (400 MHz, $\left.\mathrm{CDCl}_{3}\right): 7.16-7.31(\mathrm{~m}, 6 \mathrm{H}), 6.82-6.87(\mathrm{~m}, 4 \mathrm{H}), 6.40$ (d, $J=8.0 \mathrm{~Hz}, 1 \mathrm{H}), 4.63-4.71(\mathrm{~m}, 2 \mathrm{H}), 4.37$ (s, $2 \mathrm{H}), 3.12-3.21(\mathrm{~m}, 2 \mathrm{H}), 2.99$ (dd, $J=13.9,8.8$ $\mathrm{Hz}, 1 \mathrm{H}), 2.99(\mathrm{dd}, J=18.7,4.3 \mathrm{~Hz}, 1 \mathrm{H}), 1.38(\mathrm{~s}, 9 \mathrm{H}) .{ }^{13} \mathrm{C} \mathrm{NMR}\left(100 \mathrm{MHz}, \mathrm{CDCl}_{3}\right): \delta=206.2$, 
$168.8,168.6,154.6,129.3,128.8,127.5,115.5,115.0,114.8,83.1,73.3,55.6,54.3,48.6,41.0$, 38.2, 27.8. HRMS (CI) calcd. for $\mathrm{C}_{26} \mathrm{H}_{28} \mathrm{ClF}_{3} \mathrm{~N}_{2} \mathrm{O}_{6}[\mathrm{M}+1]^{+}:$558.1558. Found: 558.1575.

tert-Butyl 5-(4-nitrophenoxy)-4-oxo-2-((S)-3-phenyl-2-(2,2,2-trifluoroacetamido)propanamido) pentanoate (4n). Major diastereomer (68\%): ${ }^{1} \mathrm{H}$ NMR (400 MHz, $\left.\mathrm{CDCl}_{3}\right): \delta=7.13-7.31$ (m, 6 H), 6.78-6.88 (m, $4 \mathrm{H}), 6.31(\mathrm{~d}, J=7.3 \mathrm{~Hz}, 1 \mathrm{H}), 4.63-4.71(\mathrm{~m}, 2 \mathrm{H}), 4.49(\mathrm{~d}, J=16.8 \mathrm{~Hz}, 1 \mathrm{H})$, $4.45(\mathrm{~d}, J=16.9 \mathrm{~Hz}, 1 \mathrm{H}), 3.27$ (dd, $J=18.5,4.4 \mathrm{~Hz}, 1 \mathrm{H}), 3.19(\mathrm{dd}, J=13.9,6.5 \mathrm{~Hz}, 1 \mathrm{H}), 3.09$ $(\mathrm{dd}, J=13.8,6.2 \mathrm{~Hz}, 1 \mathrm{H}), 3.10-3.15(\mathrm{~m}, 1 \mathrm{H}), 1.43(\mathrm{~s}, 9 \mathrm{H}) .{ }^{13} \mathrm{C} \mathrm{NMR}\left(100 \mathrm{MHz}, \mathrm{CDCl}_{3}\right): \delta=$ 205.9, 168.8, 168.4, $156.9(J=37.5 \mathrm{~Hz}), 154.7,151.6,135.2,129.3,128.8,127.3,115.6(J=$ $285.6 \mathrm{~Hz}), 115.4$, 83.0, 73.2, 55.6, 54.7, 48.1, 40.9, 38.6, 27.7. Minor diastereomer (32\%, selected signals): ${ }^{1} \mathrm{H}$ NMR (400 MHz, $\left.\mathrm{CDCl}_{3}\right)$ : 7.14-7.29 (m, $\left.6 \mathrm{H}\right), 6.81-6.87(\mathrm{~m}, 4 \mathrm{H}), 6.40$ (d, $J=8.0 \mathrm{~Hz}, 1 \mathrm{H}), 4.63-4.71(\mathrm{~m}, 2 \mathrm{H}), 4.37$ (s, $2 \mathrm{H}), 3.12-3.21(\mathrm{~m}, 2 \mathrm{H}), 2.99$ (dd, $J=13.9,8.8$ $\mathrm{Hz}, 1 \mathrm{H}), 2.99$ (dd, $J=18.7,4.3 \mathrm{~Hz}, 1 \mathrm{H}), 1.38(\mathrm{~s}, 9 \mathrm{H}) .{ }^{13} \mathrm{C} \mathrm{NMR}\left(100 \mathrm{MHz}, \mathrm{CDCl}_{3}\right): \delta=206.2$, $168.8,168.6,154.6,129.3,128.8,127.5,115.5,115.0,114.8,83.1,73.3,55.6,54.3,48.6,41.0$, 38.2, 27.8. HRMS (CI) calcd. for $\mathrm{C}_{26} \mathrm{H}_{28} \mathrm{~F}_{3} \mathrm{~N}_{3} \mathrm{O}_{8}[\mathrm{M}+1]^{+}:$568.1862. Found: 568.1878.

\section{Acknowledgements}

This work was supported by the Deutsche Forschungsgemeinschaft and by the Fonds der Chemischen Industrie. A. F. Zahoor thanks the DAAD and HEC (Pakistan) for a PhD fellowship.

\section{References}

1. Reviews: (a) Jacobsen, E. N. Acc. Chem. Res. 2000, 33, 421. (b) Nielsen, L. P. C.; Jacobsen, E. N. In Epoxides and Aziridines in Organic Synthesis;Yudin, A. K, Ed.; Wiley-VCH: Weinheim, Germany, 2006, 229. (c) Gansäuer, A.; Justicia, J.; Fan, C. A.; Worgull, D.; Piestert, F. Top. Curr. Chem. 2007, 279, 25.

2. Recent reviews: (a) Vilotijevic, I.; Jamison, T. F. Angew. Chem. 2009, 121, 5452; Angew. Chem. Int. Ed. 2009, 48, 5250. (b) Morten, C. J.; Byers, J. A.; van Dyke, A. R.; Vilotijevic, I.; Jamison, T. F. Chem. Soc. Rev. 2009, 38, 3175, and references cited therein.

3. (a) Oka, M.; Nishiyama, Y.; Ohta, S.; Kamei, H.; Konishi, M.; Miyaki, T.; Oki, T.; Kawaguchi, H. J. Antibiot. 1988, 1331. (b) Oka, M.; Ohkuma, H.; Kamei, H.; Konishi, M.; Oki, T.; Kawaguchi, H. J. Antibiot. 1988, 1906.

4. Ohyama, T.; Kurihara, Y.; Ono, Y.; Ishikawa, T.; Miyakoshi, S.; Hamano. K.; Araei, M.; Suzuki, T.; Igari, H.; Suzuki, Y.; Inukai, M. J. Antibiot. 2000, 1108.

5. (a) Uchida, I.; Shigematsu, N.; Ezaki, M.; Hashimoto, M.; Aoki, H.; Imanaka, H. J. Antibiot. 1985, 1462. (b) Chang, C. C.; Morton, G. O.; James, C. J.; Siegel, M. M.; Kuck, N. A.; Testa, R. T.; Morders, D. B. J. Antibiot. 1991, 674. 
6. Schöllkopf, U.; Hupfeld, B.; Gull, R. Angew. Chem. 1986, 98, 755; Angew. Chem. Int. Ed. 1986, 25, 754.

7. (a) Abenhaïem, D.; Loupy, A.; Mahieu, C.; Semeria, D. Syn. Commun. 1994, 24, 1809. (b) Shiozaki, M.; Ishida, N. Chem. Lett. 1987, 1403.

8. Fox, M. E.; Lennon, I. C.; Farina, V. Tetrahedron Lett. 2007, 48, 945

9. (a) Williams, R. M.; Dung, J.-S.; Josey, J.; Armstrong, R. W.; Meyers, H. J. Am. Chem. Soc. 1983, 105, 3214. (b) El Achgar, A.; Boumzebra, M.; Roumestant, M.-L., Viallefont, P. Tetrahedron 1988, 44, 5319. (c) Fitzi, R.; Seebach, D. Tetrahedron 1988, 44, 5277. (d) Avdagic, A.; Lesac, A.; Mayer, Z.; Hollosi, M.; Sunjic, V. Helv. Chim. Acta 1998, 81, 1567.

10. Pedersen, M. L.; Berkowitz, D. B. J. Org. Chem. 1993, 58, 6966.

11. Philippe, C.; Milcent, T.; Crousse, B.; Bonnet-Delpon, D. Org. Biomol. Chem. 2009, 7, 2026.

12. (a) Kazmaier, U. Amino Acids 1996, 11, 283. (b) Deska, J.; Kazmaier U. Curr. Org. Chem. 2008, 12, 7355. (c) Kazmaier, U. In Frontiers in Asymmetric Synthesis and Application of alpha-Amino Acids; Soloshonok, V. A.; Izawa, K. Eds., ACS Books: Washington, 2009, 157 and references cited therein.

13. (a) Kazmaier, U.; Grandel, R. Synlett 1995, 945. (b) Grandel, R.; Kazmaier, U.; Nuber, B. Liebigs Ann. 1996, 1143. (c) Grandel, R.; Kazmaier, U.; Rominger F. J. Org. Chem. 1998, 63, 4524.

14. (a) Pohlman, M.; Kazmaier, U. Org. Lett. 2003, 5, 2631. (b) Pohlman, M.; Kazmaier, U.; Lindner, T. J. Org. Chem. 2004, 69, 6909. (c) Mendler, B.; Kazmaier, U. Org. Lett. 2005, 7, 1715. (d) Schmidt, C.; Kazmaier, U. Eur. J. Org. Chem. 2008, 887.

15. (a) Kazmaier, U. Current Org. Chem. 2003, 317. (b) Kazmaier, U.; Pohlman, M. Synlett, 2004, 623. (c) Kazmaier, U.; Lindner, T. Angew. Chem. 2005, 117, 3368; Angew. Chem. Int. Ed. 2005, 44, 3303. (d) Kazmaier, U.; Stolz, D. Angew. Chem. 2006, 118, 3143; Angew. Chem. Int. Ed. 2006, 45, 3072.

16. (a) Kazmaier, U.; Maier, S. J. Chem. Soc., Chem. Commun. 1998, 2535. (b) Kazmaier, U.; Maier, S. Org. Lett. 1999, 1, 1763. (c) Maier, S.; Kazmaier, U. Eur. J. Org. Chem. 2000, 1241.

17. (a) Kazmaier, U.; Maier, S. J. Org. Chem. 1999, 64, 4574. (b) Kazmaier, U.; Deska, J.; Watzke, A. Angew. Chem. 2006, 118, 4973; Angew. Chem. Int. Ed. 2006, 45, 4855. (c) Deska, J.; Kazmaier, U. Angew. Chem. 2007, 119, 4654; Angew. Chem. Int. Ed. 2007, 46, 4570. (d) Deska, J.; Kazmaier, U. Chem. Eur. J. 2007, 13, 6204.

18. Kattuboina, A.; Kaur, P.; Timmons, C.; Li. G. Org. Lett. 2006, 8, 2771.

19. Ramachandran, P. V.; Garner, G.; Pratihar, D. Org. Lett. 2007, 9, 4753.

20. (a) Greger, P. L. J. Org. Chem. 1972, 37, 1907. (b) Vader, J.; Sengers, H.; de Groot, A. Tetrahedron 1989, 45, 2131. (c) Hannesian, S.; Hodges, P. J.; Murray, P. J.; Sahoo, S. P.; J. Chem. Soc., Chem. Commun. 1986, 754.

21. (a) Van Zyl, G.; van Tamelen, E. E. J. Am. Chem. Soc. 1950, 72, 1357. (b) Bavin, P. M. G.; Hansell R. G.; Spicket W. J. Chem. Soc. 1964, 4535. (c) Dalton, L. K.; Elmes B. C. Aust. J. Chem. 1972, 25, 625. (d) Kayser, M. M.; Morand, P. Can J. Chem. 1980, 58, 302. 